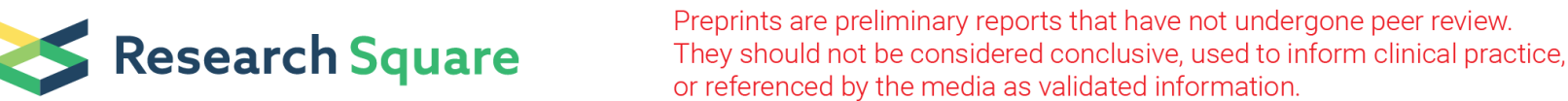

\section{8ß-Glycyrrhetinic Acid Protectes Neonatal Rats with Hyperoxia Exposure Through Inhibiting ROS/NF-KB/NLRP3 Inflammasome}

Qing Cai ( $\sim$ caiqing1982@126.com )

Pediatric Physicians' Organization at Children's https://orcid.org/0000-0002-8423-6939

\section{Ziyun Liu}

Pediatric Physicians' Organization at Children's

\section{Xuefei Yu}

Pediatric Physicians' Organization at Children's

\section{Xinyi Zhao}

Pediatric Physicians' Organization at Children's

\section{Xindong Xue}

Pediatric Physicians' Organization at Children's

Jianhua Fu

Department of Pediatrics, ShengJing Hospital of China Medical University https://orcid.org/00000002-8476-2381

\section{Research Article}

Keywords: bronchopulmonary dysplasia, inflammation, alveolarization, 18ß-Glycyrrhetinic acid, NLRP3 inflammasome, NF-kB pathway

Posted Date: November 3rd, 2021

DOI: https://doi.org/10.21203/rs.3.rs-936715/v1

License: (c) (1) This work is licensed under a Creative Commons Attribution 4.0 International License. Read Full License 


\section{Abstract}

Bronchopulmonary dysplasia (BPD) is a common devastating pulmonary complication in preterm infants. Oxygen supplementation is a lifesaving therapeutic measure used for premature infants with pulmonary insufficiency. However, oxygen toxicity is a significant trigger for BPD, and oxidative stressinduced inflammatory responses, in turn, worsens the oxidative toxicity resulting in lung injury and arresting of lung development. Glycyrrhiza radix is commonly used in the medicine and food industries. $18 \beta$-Glycyrrhetinic acid (18ß-GA), a primary active ingredient of Glycyrrhiza radix, has a powerful antioxidative and anti-inflammatory effects. This study aimed to determine whether $18 \beta-G A$ has protective effects on neonatal rats with hyperoxia exposure. Newborn Sprague-Dawley rats were kept in either $21 \%$ (normoxia) or $80 \% \mathrm{O}_{2}$ (hyperoxia) continuously from postnatal day (PN) 1 to $14.18 \beta$-GA was injected intragastrically at 50 or $100 \mathrm{mg} / \mathrm{kg}$ body weight once a day from PN 1 to 14 . We examined the body weights and alveolar development, and measured ROS level and the markers of pulmonary inflammation. Mature-IL-1 $\beta$ and NF-KB pathway proteins, and the NLRP3 inflammasome, were assessed; concurrently, caspase-1 activity was measured. Our results indicated that hyperoxia resulted in alveolar simplification and decreased bodyweight of neonatal rats. Hyperoxia exposure increased ROS level and pulmonary inflammation, and activated NF-KB and the NLRP3 inflammasome. 18ß-GA treatment decreased ROS level, inhibited the activation of NF-KB and the NLRP3 inflammasome, decreased pulmonary inflammation, improved alveolar development, and increased the bodyweight of neonatal rats with hyperoxia exposure. Our study demonstrates that 18ß-GA protects neonatal rats with hyperoxia exposure through inhibiting ROS/NF-KB/NLRP3 inflammasome.

\section{Introduction}

Bronchopulmonary dysplasia (BPD) is described as the multiple external injurious stimuli (oxygen toxicity, baro- and volu-trauma, and infection/inflammation) damage the immature, developing lung, and arrest lung development (Abman et al., 2017; Principi et al., 2018; Lignelli et al., 2019). A key histopathological feature of BPD is an alveolar simplification, which is characterized by fewer and larger alveoli (Hwang et al., 2018; Kalikkot et al., 2017). The pulmonary disfunction of BPD infants persists into adulthood, suggesting that lung injuries early in life may have lifelong consequences (Baraldi et al., 2007; Lignelli et al., 2019). BPD is no longer a disease limited to lung. BPD infants present decreased right ventricular function, increased cardiovascular risk, and higher incidence of arterial hypertension in adolescence and adulthood (Wilson-Costello et al., 2009; Lewandowski et al., 2013; Sipola-Leppänen et al., 2014; Gough et al., 2014). In addition, BPD is an independent risk factor for long-term neurodevelopmental impairment (Jensen et al., 2014; Baraldi et al., 2007; Nakanishi et al., 2016). Among 10-year-old children born extremely preterm, those who had BPD were at increased risk of cognitive and academic achievement (Sriram et al., 2018). Lowering the high incidence of BPD may be vital to improving long-term outcomes after extremely or very preterm birth. Unfortunately, current prevention strategies such as corticosteroids (Doyle et al., 2017), surfactant (Isayama et al., 2016), less invasive 
respiratory strategies (Sweet et al., 2019), careful oxygen usage (Saugstad Ola et al., 2018), and antioxidants (Poggi et al., 2014) have not been able to diminish the BPD incidence.

For the developing lung, oxygen toxicity is a significant trigger for BPD (Morty et al., 2018; Bhandari et al., 2010; Wang et al., 2018). Lung development progresses in five distinct stages: embryonic, pseudo glandular, canalicular, saccular, and alveolar (Kotecha et al., 2000). Human preterm infants who develop BPD are primarily born in the saccular stage of lung development (human 24-38 weeks of gestation age). In rodent models, the saccular stage of lung development begins at embryonic day 18 and continues through postnatal (PN) day 5 (Joshi et al., 2007). The rodent pups from birth exposed to high concentration oxygen have similar histological changes with hyperoxia-exposed human preterm infants (Nardiello et al., 2017; O'Reilly et al., 2014; Berger et al., 2014; Buczynski et al., 2013). Therefore, hyperoxia-exposure neonatal rat models are essential for better understanding the molecular mechanisms of BPD and testing the efficacy of potential therapeutic agents.

The molecular mechanisms of BPD are complex. The newborns pass from the hypoxic environment of the womb to the relatively hyperoxic extrauterine environment, and they experience increased oxidative stress. Preterm neonates are more susceptible to oxidative stress because of developmental deficits in antioxidant defenses (Kinsella et al., 2006; Negi et al., 2012). In addition, an additive oxygen therapy for the treatment of respiratory instabilities can increase oxidative stress (Perrone et al., 2016). Although premature infants are usually exposed to only the least required amount of supplemental oxygen, studies show considerable evidence of oxidant stress (Ozsurekci et al., 2016; Perrone et al., 2016). The excessive reactive oxygen species (ROS) trigger the oxidative stress response, and damage the developing lung. And oxidative stress can activate inflammatory responses, representing increased pro-inflammatory cytokines and chemokines, and inflammatory cell infiltration in lung tissue. The inflammatory cells produce more ROS to worsens the oxidative toxicity, which in turn recruits more inflammatory cells to the lung (Rosanna et al., 2012). These complex and cross-talk processes between oxidative stress and inflammation ultimately lead to lung injury and impairment of lung development, which contribute to BPD (Rosanna et al., 2012; Balany et al., 2015). However, the mechanism of the interaction between oxidative stress and inflammation has not yet been conclusively clarified.

The NLRP3 (NOD-, LRR-, and pyrin-domain containing protein 3) inflammasome is the most extensively studied inflammasome complex (Agostini et al., 2004), and NLRP3 inflammasome can be activated during oxidative stress and systemic infections (Groslambert et al., 2018; Yang et al., 2019). NLRP3 recruits ASC (apoptosis-associated Specklike protein containing CARD) and caspase-1, and format the NLRP3 inflammasome. The NLRP3 inflammasome mediates the post-translational processing of IL-1 $\beta$ by active caspase-1, which cleaves pro-IL-1 $\beta$ to produce mature IL-1 $\beta$. Mature IL-1 $\beta$ interacts with its receptor, IL1R, to signal inflammatory pathways. Activation of the NLRP3 inflammasome is considered to be a two-step process that requires two signals: the first priming signal triggers nuclear factor-KB (NF-KB)dependent upregulation of NLRP3 and pro-IL-1 $\beta$ expression (Bauernfeind et al., 2009); the second signal is to induce NLRP3 activation (Swanson et al., 2019; Yang et al., 2019). ROS has been considered as a trigger of NLRP3 inflammasome activation (Teng et al., 2020; Wei et al., 2020), and many chemical 
compounds are reported to activate NLRP3 inflammasome via increasing intracellular ROS levels (Zhong et al., 2013). In addition, ROS can activate the NF-KB signaling pathway, resulting in the amplification of inflammatory response (Zha et al., 2014). ROS-mediated NF-KB signaling plays an essential role in activating the NLRP3 inflammasome (Teng et al., 2020; Peng et al., 2020). Therefore, ROS/NF-KB/NLRP3 inflammasome signaling is closely associated with the overlapping or feedback pathways between oxidative stress and inflammatory response.

Caffeine is a kind of plant alkaloid, and exhibits practical benefit in the treatment or prevention of BPD in several clinical studies (Coulter et al., 2006; Sanchez-Solis et al., 2020; Pakvasa et al., 2018). Endesfelder et al. reported that Caffeine prevented oxygen-induced inflammatory lung injury in neonatal rats (Endesfelder et al., 2020). Chen et al. reported that Caffeine treatment could protect hyperoxia-induced mice lung from oxidative damage by inhibiting NLRP3 inflammasome and NF-KB pathway (Chen et al., 2020). Besides Caffeine, others plant-derived compounds are beneficial to human health. Glycyrrhiza radix is one of the most popular phytochemicals, widely used in the medicine and food industries (Pastorino et al., 2018; El-Saber et al., 2020). Triterpene glycoside 18ß-glycyrrhetinic acid (18ß-GA), extracted from Glycyrrhiza radix, is a promising bioactive compound with anti-oxidative and antiinflammatory properties (Pastorino et al., 2018; El-Saber et al., 2020). Animal studies have confirmed that $18 \beta$-GA has a protective effect against pulmonary diseases, such as asthma, pulmonary hypertension, pulmonary infection, and pulmonary fibrosis (Kim et al., 2017; Zhang et al., 2019; Chen et al., 2017; Kao et al., 2010). All of these studies have reflected that 18ß-GA has beneficial effects on lungs.

Based on these considerations, we hypothesized that 18ß-GA had a protective effect in neonatal rats with hyperoxia exposure. In this study, we investigated the role of ROS/NF-KB/NLRP3 inflammasome in this process.

\section{Materials And Methods 2.1 Animals}

Pregnant Sprague-Dawley (SD) rats were purchased from the Department of Animals, Experimental Center, Shengjing Hospital of China Medical University (Shenyang, China). All newborn SD rats were born on days 21-23 of gestation. Both male and female neonatal rats were used in all studies. Each maternal rat was adjusted to feed 6-8 pups to minimize nutritional differences on lung development. Maternal rats were rotated between room air and oxygen-exposed litters daily to prevent oxygen toxicity. The rat's living environment was as follows: $12 \mathrm{~h}$ alternating light/dark cycle, the temperature at $25^{\circ} \mathrm{C}-26^{\circ} \mathrm{C}$, and humidity at $60 \%-70 \%$. The chamber was opened once per day for $0.5 \mathrm{~h}$ to replace the food and water. All animal procedures were approved by the Laboratory Animal Ethics Committee of Shengjing Hospital of China Medical University (Shenyang, China).

\subsection{Animal experimentation: oxygen exposure and 18 $\beta-G A$ treatment}


Neonatal rats were exposed to $21 \% \mathrm{O}_{2}$ (normoxia) or $80 \% \mathrm{O}_{2}$ (hyperoxia) from postnatal day (PN) 1 to 14 . A total of one hundred neonatal rats were randomly divided into five groups: normoxia group ("N group," $\mathrm{n}=20$ ), normoxia and $100 \mathrm{mg} / \mathrm{kg} / \mathrm{d}$ 18ß-GA group ("N + 18ß-GA 100 group," $\mathrm{n}=20$ ), hyperoxia group ("H

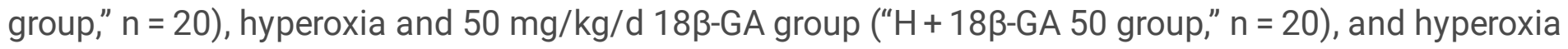
and $100 \mathrm{mg} / \mathrm{kg} / \mathrm{d}$ 18 $\beta$-GA group (" $\mathrm{H}+18 \beta-\mathrm{GA} 100$ group," $\mathrm{n}=20$ ). The concentration of $\mathrm{CO}_{2}$ was

maintained at $<0.5 \%$ by absorbing excess $\mathrm{CO}_{2}$ with soda lime. Sixty neonatal rats were treated with $18 \beta$ GA (G10105, Sigma, Carlsbad, CA) via intragastrical injection at 50 or $100 \mathrm{mg} / \mathrm{kg}$ body weight once daily from PN1 to 14. 18ß-GA was dissolved in saline, and administration volume was $10 \mathrm{ul} / 10 \mathrm{~g}$ bodyweight of rats. The purity of $18 \beta-G A$ was measured at $97 \%$ by HPLC. The molecular formula for $18 \beta-G A$ is $\mathrm{C}_{30} \mathrm{H}_{46} \mathrm{O}_{4}$, and the molecular weight is 470.68 (Fig. $1 \mathrm{~A}$ ).

\subsection{Bodyweight measurement and lung sample collection}

The bodyweights of all neonatal rats were measured at PN 14, after which they were sacrificed by an intraperitoneal injection of pentobarbital ( $50 \mathrm{mg} / \mathrm{kg}$ body weight). BALF was centrifuged at $500 \mathrm{~g}$ for 10 min immediately after extraction. The BALF supernatant was frozen at $-80^{\circ} \mathrm{C}$ until further analysis. The pulmonary artery was perfused with PBS to remove all blood from the lungs. The lungs for the molecular analysis were immediately frozen in liquid nitrogen and stored at $-80^{\circ} \mathrm{C}$. For immunohistochemical analysis, the lungs were inflated to $25 \mathrm{~cm} \mathrm{H}_{2} \mathrm{O}$ with $4 \%$ paraformaldehyde (PFA), then fully fixed in $4 \%$ PFA for $48 \mathrm{~h}$ and embedded in paraffin.

\subsection{Lung histological and morphometric analyses}

Inflation-fixed lungs were processed to obtain $3 \mu \mathrm{m}$ thick paraffin sections, stained with hematoxylin and eosin for examination of the lung architecture. Six images were randomly selected for each sample. Alveolarization was evaluated by the radial alveolar count (RAC) value (Cooney et al., 1982) which was obtained by drawing a line from the center of terminal bronchioles to the nearest connective tissue septum and counting the number of the alveoli on the line. These assessments were carried out independently by two pathologists who were blinded to the grouping.

\subsection{Inflammatory cells in BALF and Giemsa staining}

BALF supernatant cell smears were examined using Giemsa staining (D010-1-1, Nanjing Jiancheng Research Institute of Biotechnology, Jiangsu, China) according to the manufacturer's instructions. The inflammatory cells were counted and classified under a light microscope.

\subsection{Pro-inflammatory cytokines in BALF and ELISA}

The expression of IL-1 $\beta$, IL-6, and tumor necrosis factor (TNF)- $a$ in BALF was analyzed using enzymelinked immunosorbent assay (ELISA) kits (EK301BHS, EK306HS, EK382HS, Multi Sciences, China) according to the manufacturer's instructions.

\subsection{ROS Measurement}


We centrifugated the homogenates of lung tissues at $10,000 \mathrm{~g}$ for $15 \mathrm{~min}$ at $4^{\circ} \mathrm{C}$ and collected the supernatant for subsequent testing. ROS level was assayed by a ROS assay kit (WanLeiBio, China), which used a stable non-fluorescent dichlorodihydrofluorescein diacetate (DCFH-DA) as a probe. DCFH-DA freely entered cells and was then hydrolyzed by esterases to create non-fluorescent DCFH. However, DCFH was rapidly oxidized by ROS in the cells to generate strong fluorescent DCF. Hence, ROS levels were assayed indirectly via measuring DCF fluorescence.

\subsection{Myeloperoxidase and N-Acetyl- $\beta$-D-glucosaminidase activity assays in lung tissues}

The neutrophils and macrophages in lung homogenates were analyzed by measuring myeloperoxidase (MPO) activity, using an MPO assay kit (A044-1-1, Nanjing Jiancheng Research Institute of Biotechnology, Jiangsu, China), and N-Acetyl- $\beta$-D-glucosaminidase (NAG) activity, using a NAG assay kit (A031, Nanjing Jiancheng Research Institute of Biotechnology, Jiangsu, China), respectively, according to the manufacturers' instructions.

\subsection{Caspase-1 activity assay in lung tissues}

Caspase-1 activity in lung homogenates was analyzed with a caspase-1 activity assay kit (C1101, Beyotime, China) according to the manufacturer's instructions.

\subsection{Western blotting}

The lung tissue samples were homogenized in RIPA lysis buffer with a protease inhibitor (P1045, Beyotime Institute of Biotechnology, China). The protein concentration was measured using the bicinchoninic acid method, and samples were boiled with a loading buffer for protein extraction. In total, $40 \mu \mathrm{g} /$ lane samples were loaded onto a $4 \%-20 \%$ gel, resolved using SDS-PAGE, and subsequently transferred to polyvinylidene fluoride (PVDF) membranes. The PVDF membranes were then blocked at room temperature using $5 \%$ skimmed milk for $2 \mathrm{~h}$ to prevent nonspecific binding. The following primary antibodies were used: mature IL-1 $\beta$ (1:500, WL00891, Wanleibio, China); cleaved caspase-1/caspase-1 (1:500, WL02996, Wanleibio, China); NLRP3 (1:1000, WL02635, Wanleibio, China); ASC (1:1000, bs6741R, Bioss, China); NF-KB (1:500, WL01980, Wanleibio, China); p-NF-KB (1:500, WL02169, Wanleibio, China); IkBa (1:500, WL01936, Wanleibio, China); p-IkBa (1:500, WL02495, Wanleibio, China); $\beta$-actin (1:1000, WL01372, Wanleibio, China). The PVDF membranes were incubated with the primary antibodies, diluted in 5\% skimmed milk in tris-buffered saline and Tween20 (TBST), overnight at $4^{\circ} \mathrm{C}$. The next day, the PVDF membranes were washed three times with TBST and incubated with the appropriate secondary antibody (goat anti-rabbit IgG-HRP, 1:5000, WLA023, Wanleibio, China) at room temperature for $2 \mathrm{~h}$, after which they were rewashed with TBST again. The protein bands were detected using an enhanced chemiluminescence substrate kit (PK10001, Proteintech, China). The optical density of the target strip was analyzed by Gel-Pro-Analyzer software.

\subsection{Real-Time PCR}


Lung tissues were cut in Trizol (9108, Takara, Japan) to isolate and purify the nucleic acid. The A260/A280 ratio was used to adjust the RNA concentration. Primescript RT (Takara, Japan) and SYBR Premier Ex Taq II (Takara, Japan) kits were used for reverse transcription and amplification, respectively. Detection was performed using a Roche LC480 Light Cycler with the following primers: MIF: forward:5'GCAAGCCGGCACAGTACAT-3', reverse:5'-GCTCGTGCCACTAAAA GTCATG-3';MIP-2ロforward:5'CCTACCAAGGGTTGACTTCAAGA-3', reverse: 5'-GCTTCAGGGTTGAGACAAACTTC-3'םCINC-10forward:5'GCTGTCAGTGCC TGCAGACA-3', reverse: 5'-GACCATTCTTGAGTGTGGCTATGA-3' $\beta$-actin: forward: 5'CGTGCGTGACATTAAAGAG-3', reverse: 5'-TTGC CGATAGTGATG ACCT-3'. The relative amount of transcripts was calculated using the $2(-\triangle \Delta \mathrm{Ct})$ method, normalized to the $\beta$-actin transcript as an internal control.

\subsection{Statistical analysis}

Data analysis was performed using GraphPad Prism version 8.0 (GraphPad Software). Experimental data were presented as the mean \pm standard deviation. Comparisons among multiple groups were conducted using one-way analysis of variance (ANOVA) followed by Tukey's post hoc test (equal variance) or Dunnett T3's post hoc test (unequal variance). $P<0.05$ was considered statistically significant.

\section{Results}

\subsection{Effect of 18ß-GA on bodyweight of neonatal rats with hyperoxia exposure}

The bodyweights of neonatal rats in the different groups were measured at PN14. Exposure to $80 \% \mathrm{O}_{2}$ resulted in a decrease in the bodyweights of neonatal rats $(P<0.01$; Fig. 1B). However, treatment with 18ß-GA increased the bodyweights of neonatal rats exposed to $80 \% \mathrm{O}_{2}(P<0.01$; Fig. 1B).

\subsection{8ß-GA increased alveolarization of neonatal rats with hyperoxia exposure}

The neonatal rats exposed to $21 \% \mathrm{O}_{2}$ had a standard distal lung architecture with well-formed alveoli and a standard RAC value (Fig. 2). However, distal lung histology in neonatal rats exposed to $80 \% \mathrm{O}_{2}$ showed simplification of the distal lung architecture, with fewer, larger alveoli and a lower RAC value $(P<0.01$; Fig. 2). The hyperoxia exposed and 18ß-GA-treated rats had increased alveolarization and a higher RAC value than the rats with hyperoxia exposure alone $(P<0.01 ;$ Fig. 2$)$. These results demonstrated that hyperoxia exposure resulted in alveolar simplification; however, $18 \beta-G A$ treatment protected neonatal rats with hyperoxia exposure from alveolar simplification.

\subsection{8ß-GA decreased pulmonary inflammation of neonatal rats with hyperoxia exposure}


We detected inflammatory cells in the BALF of neonatal rats in different groups. Neonatal rats exposed to $80 \% \mathrm{O}_{2}$ had a significantly elevated inflammatory cell count in their BALF, especially neutrophils and macrophages $(P<0.05$; Table 1). Treatment with $18 \beta$-GA decreased neutrophil and macrophage count in the BALF of neonatal rats exposed to $80 \% \mathrm{O}_{2}(P<0.05$; Table 1$)$. Further, we investigated neutrophils and macrophages infiltration in lung tissues, by measuring the specific enzyme activities of MPO and NAG in lung homogenates. MPO and NAG activities increased in rats exposed to $80 \% \mathrm{O}_{2}(P<0.01$; Fig. 3A-B). The increased MPO and NAG activities were reversed by $18 \beta-$ GA treatment $(P<0.01 ;$ Fig. 3A-B). These results suggest that $18 \beta-G A$ treatment halts the inflammatory cell infiltration caused by hyperoxia exposure.

Table 1

Classification and counting of inflammatory cells in BALF $\left(\times 10^{3}\right.$ cells $/ \mathrm{ml}$, mean $\left.\pm \mathrm{SD}\right)$

\begin{tabular}{|c|c|c|c|c|c|}
\hline Groups & Total cells & Neutrophils & Macrophages & Lymphocytes & Eosinophils \\
\hline $\mathbf{N}$ & $90.67 \pm 3.77$ & $1.86 \pm 0.14$ & $82.77 \pm 5.20$ & $4.63 \pm 0.40$ & $1.58 \pm 0.10$ \\
\hline $\begin{array}{l}N+18 \beta-G A \\
100\end{array}$ & $\begin{array}{l}94.17 \pm 5.36 \\
\mathrm{NS}\end{array}$ & $2.03 \pm 0.13 \mathrm{NS}$ & $\begin{array}{l}85.46 \pm 8.62 \\
\text { NS }\end{array}$ & $\begin{array}{l}4.45 \pm 0.42 \\
\text { NS }\end{array}$ & $\begin{array}{l}1.66 \pm 0.16 \\
\text { NS }\end{array}$ \\
\hline H & $\begin{array}{l}289.17 \pm \\
27.45^{\star \star}\end{array}$ & $125.46 \pm 12.37$ & $\begin{array}{l}129.88 \pm \\
6.26 * \star\end{array}$ & $6.96 \pm 0.41^{\star * *}$ & $\begin{array}{l}13.21 \pm \\
1.04^{\star \star}\end{array}$ \\
\hline $\begin{array}{l}H+18 \beta-G A \\
50\end{array}$ & $\begin{array}{l}264.17 \pm 23.17 \\
\text { NS }\end{array}$ & $76.42 \pm 6.16 \# \#$ & $\begin{array}{l}118.83 \pm 9.68 \\
\text { NS }\end{array}$ & $\begin{array}{l}6.33 \pm 0.17 \\
\text { NS }\end{array}$ & $\begin{array}{l}12.25 \pm 1.14 \\
\text { NS }\end{array}$ \\
\hline $\begin{array}{l}H+18 \beta-G A \\
100\end{array}$ & $\begin{array}{l}171.67 \pm \\
5.53 \# \#\end{array}$ & & $\begin{array}{l}84.13 \pm \\
4.17 \# \#\end{array}$ & $\begin{array}{l}4.46 \pm \\
0.27 \# \#\end{array}$ & $\begin{array}{l}5.52 \pm \\
0.44 \# \#\end{array}$ \\
\hline
\end{tabular}

Chemokines play an essential role in mediating inflammatory cell recruitment to the lung during hyperoxic exposure (Endesfelder et al., 2020). As shown in Fig. 4A-C, we observed a robust increase in the expression of the cytokine-induced neutrophil chemoattractant-1 (CINC-1) $(P<0.01$;Fig. 4A), macrophage inflammatory protein-2 (MIP-2) $(P<0.01$;Fig. 4B), and macrophage migration inhibitory factor (MIF) $(P<$ 0.01 ;Fig. 4 C) in the hyperoxia group compared to the normoxic group, which was alleviated by $18 \beta-G A$ treatment.

Next, we measured the pro-inflammatory cytokines levels in the BALF. Rats exposed to $80 \% \mathrm{O}_{2}$ had increased expression of IL-1 $\beta$, IL-6, and TNF- $\alpha$ in their BALF ( $P<0.01$; Fig. 5A-C). 18ß-GA treatment reversed the increase in pro-inflammatory cytokines expression under hyperoxic conditions $(P<0.01$; Fig. $5 A-C)$. These results suggest that $18 \beta-G A$ treatment reduces the inflammatory response caused by hyperoxia exposure.

\subsection{8 $\beta$-GA decreased pulmonary ROS level of neonatal rats with hyperoxia exposure}


As depicted in Fig. 6 showed that rats exposed to $80 \% \mathrm{O}_{2}$ had a significantly elevated ROS level in the lung tissues. Treatment with 18ß-GA reversed the increase in ROS level under hyperoxic conditions $(P<$ $0.01)$.

\subsection{Treatment with 18ß-GA inhibited the activity of NF-KB and the NLRP3 inflammasome and decreased mature-IL-1 $\beta$ levels}

We investigated whether $18 \beta-\mathrm{GA}$ treatment inhibited the activity of the NF-kB pathway using western blot analysis. As shown in Fig. 7, phosphor-NF-KB/NF-kB $(P<0.01)$ and phosphor-lkBa/lkBa protein relative expression $(P<0.01)$ were significantly increased in rats exposed to $80 \% \mathrm{O}_{2}$, and $18 \beta$-GA treatment reversed these changes $(P<0.01)$. These results indicated that 18 $\beta$-GA treatment inhibited the activation of the NF-KB pathway in rats exposed to hyperoxia.

Next, we tested the effect of $18 \beta-G A$ on the NLRP3 inflammasome. As shown in Fig. 8A-C, rats exposed to $80 \% \mathrm{O}_{2}$ had increased expression of NLRP3, ASC, and cleaved caspase-1 $(P<0.01)$, reversed by $18 \beta-\mathrm{GA}$ treatment. Furthermore, we found that rats exposed to $80 \% \mathrm{O}_{2}$ had increased caspase- 1 activity which

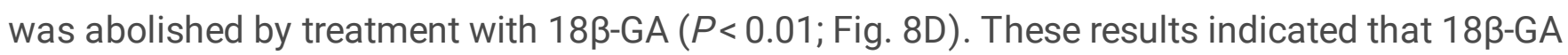
treatment inhibited the increased activity of NLRP3 inflammasome induced by hyperoxia exposure.

Since hyperoxia exposure increased the expression of cleaved caspase-1, we further tested whether hyperoxia exposure could increase the level of mature IL-1 $\beta$. Similarly, with cleaved caspase-1, rats exposed to $80 \% \mathrm{O}_{2}$ had increased expression of mature IL-1 $\beta$, and $18 \beta$-GA-treated rats had a lower protein expression of mature IL-1 $\beta(P<0.01$; Fig. 9). Based on the above findings, $18 \beta-$ GA treatment downregulated the increased expression of mature-IL-1 $\beta$ induced by hyperoxia exposure, through inhibiting the activity of the NLRP3 inflammasome.

\section{Discussion}

In this study, we exposed neonatal rats in $80 \% \mathrm{O}_{2}$ from birth to PN 14 , and $18 \beta$-GA treatment was concurrently applied. Our results show that 18ß-GA treatment increased bodyweight and improved alveolar simplification of neonatal rats with hyperoxia exposure. Under hyperoxia conditions, 18ß-GA treatment decreased ROS level and inflammation response, and inhibited the activity of NF-KB and the NLRP3 inflammasome. Our results indicated that 18ß-GA treatment protected neonatal rats undergoing hyperoxia exposure from alveolar simplification by inhibiting ROS/NF-KB/NLRP3 inflammasome.

Advances in the management of preterm infants over the past 50 years, the survival rate of extremely and very premature infants has improved. In contrast, the incidence of BPD is almost unchanged (Stoll et al., 2015). The overall incidence of BPD in infants born at $<28$ weeks of gestational age is estimated to be $48 \%-68 \%$ (Stoll et al., 2010). To date, no adequate or preventive therapy of BPD is available. $18 \beta-G A$, as an anti-oxidative and anti-inflammatory drug, has been proved to play a protective role in pulmonary diseases (Kim et al., 2017; Zhang et al., 2019; Chen et al., 2017; Kao et al., 2010). In a mouse model of 
allergic asthma (Kim et al., 2017), 18ß-GA strongly suppressed airway hyperresponsiveness, accumulation of inflammatory cells, and levels of T helper type 2 (Th2) cytokines in BALF. Zhang et al. reported $18 \beta-G A$ had a protective effect against monocrotaline-induced pulmonary arterial hypertension by inhibiting oxidative stress in rats (Zhang et al., 2019). In a mouse model of radiation-induced lung injury, GA administration mitigated the histologic changes of lung injury by inhibiting the TGF- $\beta 1 /$ Smads signaling pathway (Chen et al., 2017). In vitro, 18ß-GA reduced inflammatory cytokine production in the RAW264.7 cells (macrophage of BALB/c mice) treated with LPS (Kao et al., 2010). Therefore, we speculate that 18ß-GA may be used as an anti-oxidative and anti-inflammatory drug for the prevention and, or treatment of BPD.

Hyperoxia exposure animal models have been reported to mimic human BPD (Nardiello et al., 2017; O'Reilly et al., 2014; Berger et al., 2014; Buczynski et al., 2013). We exposed the neonatal rats to $80 \% \mathrm{O}_{2}$ from birth to PN 14, which represented the saccular and alveolar stage in rats lung development. In our study, we demonstrated that compared with rats exposed to $21 \% \mathrm{O}_{2}$, rats exposed to $80 \% \mathrm{O}_{2}$ had decreased bodyweight and alveolar simplification with a lower RAC value. And these results were similar to that in Chen's study (Chen et al., 2020). Our results indicated that 18ß-GA treatment in hyperoxia exposed rats increased weight gain, and improved alveolarization, as demonstrated by a higher RAC value. The above data showed that 18ß-GA treatment had a protective effect in hyperoxia-exposed neonatal rats.

Exposure to high levels of oxygen inevitably leads to the production and accumulation of excessive ROS and activation of inflammatory response, and the inflammatory reaction triggered by hyperoxia, in turn, worsens the oxidative toxicity. Pulmonary inflammation of BPD is characterized by chemotactic factors, inflammatory cells, and pro-inflammatory cytokines (Savani et al., 2018; Balany et al., 2015; Ryan et al., 2008). The influx of inflammatory cells (neutrophils and monocytes) into the lung is mediated by chemotactic factors such as CINC-1, MIP-2, and MIF, which are highly elevated in hyperoxic lungs (Bhatia et al., 2012). The influx of inflammatory cells increased release and production of pro-inflammatory cytokines (IL-1 $\beta$, IL-6, and TNF-a), which aggravate hyperoxia-induced lung injury. Bry et al. reported that mature IL-1 $\beta$ overexpression in lung epithelial cells enhances the production of neutrophil- and macrophage-attractant chemokines, causing an influx of neutrophils and macrophages, thereby disrupting the formation of the secondary septum, leading to simplification of alveoli (Bry et al., 2007). Liao et al. reported that blockade of IL-1 $\beta$ results in decreased inflammation and better alveolarization (Liao et al., 2015). Endesfelder et al. demonstrated Caffeine, as an anti-oxidative and anti-inflammatory drug, prevented neonatal rats from oxygen-induced inflammatory lung Injury (Endesfelder et al., 2020). Our study found that ROS level was significantly increased in neonatal rats with hyperoxic exposure. Treatment with $18 \beta-G A$ decreased the ROS level of rats under hyperoxic conditions. And we confirmed that chemotactic factors CINC-1, MIP-2, and MIF were significantly increased in the rats with hyperoxia exposure; a large number of neutrophils and macrophages and pro-inflammatory cytokines (IL-1 $\beta$, IL- 6 , and TNF-a) moved into the BALF of rats exposed to hyperoxia. We also found that hyperoxia exposure increased MPO and NAG activities in lung tissues, indicating that hyperoxia causes a large influx of 
neutrophils and macrophages into the lung tissues of rats. However, the influx of chemotactic factors, inflammatory cells, and pro-inflammatory cytokines was reversed by 18ß-GA treatment. Based on the above findings, our results indicated that $18 \beta-G A$, as a kind of free radical scavenger, inhibited the inflammatory response induced by hyperoxia.

ROS/NF-KB/NLRP3 inflammasome signaling may be a link between oxidative stress and inflammatory response. ROS acts as the priming signal to activate the NLRP3 inflammasome and then promote the secretion of mature IL-1 $\beta$ (Teng et al., 2020; Wei et al., 2020). In an in vitro model of non-small cell lung cancer, Teng et al. demonstrated that N-acetyl-L-cysteine (NAC), a scavenger of ROS, remarkably inhibited the cell death, and the activation of NF-KB and the NLRP3 inflammasome in the A549 and H1299 cells (Teng et al., 2020). NF-KB, a ubiquitous transcription factor, promotes the expression of many genes, including pro-inflammatory cytokines associated with the development of BPD (Wright et al., 2011). NF$\mathrm{kB}$ concentrations in cells from tracheal lavages of premature infants on the 3rd day after birth are higher in those who go on to develop BPD (Bourbia et al., 2006). Agents that inhibit NF-kB activation have shown promise in clinical trials to prevent BPD (Aghai et al., 2006; Aghai et al., 2007; Wright et al., 2010). Another study showed that hyperoxia exposure impaired alveolarization of neonatal mice by activiting NLRP3 inflammasome (Liao et al., 2015). In the present study, we confirmed that the hyperoxia exposure increased ROS level, activated NF-KB and the NLRP3 inflammasome, and increased the expression level of mature IL-1 $\beta$. However, $18 \beta-G A$ treatment reversed these effects. Therefore, our results indicated that $18 \beta-G A$ protected neonatal rats with hyperoxia exposure through inhibiting ROS/NF-KB/NLRP3 inflammasome.

\section{Limitations}

BPD is a complex multifactorial disease, including oxygen toxicity, perinatal inflammation, perinatal hypoxia, and mechanical ventilation (Nardiello et al., 2017; O'Reilly et al., 2014; Berger et al., 2014; Buczynski et al., 2013). Hyperoxia exposure is only one of the known risk factors for BPD. In addition, researchers performed the hyperoxia exposure rodent models with various oxygen concentrations ( 40 to $100 \% \mathrm{O}_{2}$ ) and duration of oxygen exposure (3 to 28 days) for different severity degrees and consequences of lungs (Nardiello et al., 2017; O'Reilly et al., 2014; Berger et al., 2014; Buczynski et al., 2013). This study exposed neonatal rats with $80 \% \mathrm{O}_{2}$ for 14 days, and future studies are needed to investigate the protective role of 18ß-GA in neonatal rats exposed to different oxygen concentrations and, or duration of oxygen exposure, even other risk factors of BPD.

\section{Conclusion}

In summary, our study suggested that $18 \beta$-GA treatment protected neonatal rats with hyperoxia exposure. The possible mechanism is that $18 \beta-G A$, as a kind of free radical scavenger, decreased the hyperoxiainduced inflammatory response through inhibiting NF-KB/NLRP3 inflammasome signaling.

\section{Declarations}


Acknowledgments

The authors would like to thank Professor Dongyan Liu from the Department of Immunology, Shengjing Hospital of China Medical University, for providing technical support.

\section{Funding}

This study was supported by grants from The National Natural Science Foundation of China (grant no. 81571479) and the 345 Talent Project of the Shengjing Hospital (grant no. M0428).

\section{Availability of data and materials}

The datasets used and, or analyzed during the current study are available from the corresponding author on reasonable request.

\section{Authors' contributions}

$\mathrm{QC}, \mathrm{XX}$ and JF conceived and designed the study. QC, ZL, $X Z$, and $X Y$ performed the experiments. QC, ZL, $X Z$, and $X Y$ analysed the data and JF reviewed the data. QC wrote the manuscript. All authors read and approved the final manuscript.

\section{Ethics approval and consent to participate}

The present study was approved by The Ethics committee of China Medical University (Approval No.: 2021PS267K).

\section{Competing interests}

The authors declare that they have no competing interests.

\section{Consent for Publication}

Not applicable.

\section{References}

1. Abman, S. H., E. Bancalari, and A. Jobe. 2017. The evolution of bronchopulmonary dysplasia after 50 years. Am J Respir Crit Care Med 195: 421-424. DOI:10.1164/rccm.201611-2386ED.

2. Aghai, Z. H., S. Kumar, S. Farhath, M. A. Kumar, J. Saslow, T. Nakhla, R. Eydelman, L. Strande, G. Stahl, C. Hewitt, M. Nesin, and I. Rahman. 2006. Dexamethasone suppresses expression of nuclear factor-kB in the cells of tracheobronchial lavage fluid in premature neonates with respiratory distress. Pediatr Res 59 (6): 811-815. DOI:10.1203/01.pdr.0000219120.92049.b3.

3. Aghai, Z. H., A. Kode, J. G. Saslow, T. Nakhla, S. Farhath, G. E. Stahl, R. Eydelman, L. Strande, P. Leone, and I. Rahman. 2007. Azithromycin suppresses activation of nuclear factor-kB and synthesis 
of proinflammatory cytokines in tracheal aspirate cells from premature infants. Pediatr Res 62 (4): 483-488. DOI:10.1203/PDR.0b013e318142582d.

4. Agostini, L., F. Martinon, K. Burns, M. F. McDermott, P. N. Hawkins, and J. Tschopp. 2004. NALP3 forms an IL-1beta-processing inflammasome with increased activity in Muckle-Wells autoinflammatory disorder. Immunity 20 (3): 319-325. DOI:10.1016/s1074-7613(04)00046-9.

5. Balany, J., and V. Bhandari. 2015. Understanding the Impact of Infection, Inflammation, and Their Persistence in the Pathogenesis of Bronchopulmonary Dysplasia. Front Med 2: 90. DOI:10.3389/fmed.2015.00090.

6. Baraldi, E., and M. Filippone. 2007. Chronic lung disease after premature birth. N Engl J Med 357: 1946-1955. DOI:10.1056/NEJMra067279.

7. Bauernfeind, F. G., G. Horvath, A. Stutz, E. S. Alnemri, K. MacDonald, and D. Speert, et al. 2009. Cutting Edge: NF-KB activating pattern recognition and cytokine receptors license NLRP3 inflammasome activation by regulating NLRP3 expression. J Immunol 183: 787-791. DOI:10.4049/jimmunol.0901363.

8. Berger, J., and V. Bhandari. 2014. Animal models of bronchopulmonary dysplasia. The term mouse models. Am J Physiol Lung Cell Mol Physio/ 307: L936-L947. DOl:10.1152 /ajplung. 00159.2014.

9. Bhandari, V. 2010. Hyperoxia-derived lung damage in preterm infants. Semin Fetal Neonatal Med 15 (4): 223-229. DOI:10.1016/j.siny.2010.03.009.

10. Bhatia, M., R. L. Zemans, and S. Jeyaseelan. 2012. Role of chemokines in the pathogenesis of acute lung injury. Am J Respir Cell Mol Biol 46 (5): 566-572. DOI:10.1165/rcmb.2011-0392TR.

11. Bourbia, A., M. A. Cruz, and H. J. Rozycki. 2006. NF-kappaB in tracheal lavage fluid from intubated premature infants: association with inflammation, oxygen, and outcome. Arch Dis Child Fetal Neonatal Ed 91 (1): F36-F39. DOI:10.1136/adc.2003.045807.

12. Bry, K., J. A. Whitsett, and U. Lappalainen. 2007. IL-1 beta disrupts postnatal lung morphogenesis in the mouse. Am J Respir Cell Mol Bio/36: 32-42. DOI:10.1165/rcmb.2006-01160C.

13. Buczynski, B. W., E. T. Maduekwe, and M. A. O'Reilly. 2013. The role of hyperoxia in the pathogenesis of experimental BPD. Semin Perinatol 37 (2): 69-78. DOI:10.1053/j.semperi.2013.01.002.

14. Chen, J., W. Zhang, L. Zhang, J. Zhang, X. Chen, M. Yang, T. Chen, and J. Hong. 2017. Glycyrrhetinic acid alleviates radiation-induced lung injury in mice. J Radiat Res 58: 41-47.

DOI:10.1093/jrr/rrw091.

15. Chen, S., Q. Wu, D. Zhong, C. Li, and L. Du. 2020. Caffeine prevents hyperoxia-induced lung injury in neonatal mice through NLRP3 inflammasome and NF-KB pathway. Respir Res 21: 140.

DOI:10.1186/s12931-020-01403-2.

16. Cooney, T. P., and W. M. Thurlbeck. 1982. The radial alveolar count method of Emery and Mithal: a reappraisal 2-intrauterine and early postnatal lung growth. Thorax 37: 580-583.

DOI:10.1136/thx.37.8.580.

17. Coulter, D. M.: Caffeine for apnea of prematurity. N Engl J Med, 2006, 355(9), 958-9; author reply 959 - 60. DOI:10.1056/NEJMc061615. 
18. Doyle, L. W., J. L. Cheong, R. A. Ehrenkranz, and H. L. Halliday. 2017. Early (< 8 days) systemic postnatal corticosteroids for prevention of bronchopulmonary dysplasia in preterm infants. Cochrane Database Syst Rev 10 (undefined): CD001146. DOI:10.1002/14651858.CD001146.pub5.

19. El-Saber, B. G., B. A. Magdy, A. El-Mleeh, M. M. Abdel-Daim, and H. Prasad Devkota: Glycyrrhiza glabra Traditional Uses, Bioactive Chemical Constituents, and Pharmacological and Toxicological Activities of L. (Fabaceae). Biomolecules. 2020. 10 (3), undefined. DOI:10.3390/ biom10030352.

20. Endesfelder, S., E. Strauß, I. Bendix, T. Schmitz, and C. Bührer: Prevention of Oxygen-Induced Inflammatory Lung Injury by Caffeine in Neonatal Rats. Oxid Med Cell Longev, 2020 (undefined), 3840124. DOI:10.1155/2020/3840124.

21. Gough, A., M. Linden, D. Spence, C. C. Patterson, H. L. Halliday, and L. P. A. McGarvey. 2014. Impaired lung function and health status in adult survivors of bronchopulmonary dysplasia. Eur Respir $\mathrm{J} 43$ (3): 808-816. DOI:10.1183/09031936.00039513.

22. Groslambert, M., and B. F. Py. 2018. Spotlight on the NLRP3 inflammasome pathway. J Inflamm Res 11 (undefined): 359-374. DOI:10.2147/JIR.S141220.

23. Hwang, J. S., and V. K. Rehan: Recent advances in Bronchopulmonary dysplasia: pathophysiology, prevention, and treatment. Lung,2018,196: 129-138. DOI:10.1007/s00408-018-0084-z.

24. Isayama, T., H. Iwami, S. McDonald, and J. Beyene. 2016. Association of Noninvasive Ventilation Strategies With Mortality and Bronchopulmonary Dysplasia Among Preterm Infants: A Systematic Review and Meta-analysis. JAMA316 (6): 611-624. DOI:10.1001/jama.2016.10708.

25. Kalikkot, T. R., M. Guaman, and B. Shivanna: Bronchopulmonary dysplasia: a review of pathogenesis and pathophysiology. Respir Med,2017,132: 170-177. DOI: 10.1016/j. rmed. 2017. 10.014.

26. Kao, T. C., M. H. Shyu, and G. C. Yen. 2010. Glycyrrhizic acid and 18beta-glycyrrhetinic acid inhibit inflammation via PI3K/Akt/GSK3beta signaling and glucocorticoid receptor activation. J Agric Food Chem 58: 8623-8629. DOI:10.1021/jf101841r.

27. Kim, S. H., J. H. Hong, J. E. Lee, and Y. C. Lee. 2017. 18ß-Glycyrrhetinic acid, the major bioactive component of Glycyrrhizae Radix, attenuates airway inflammation by modulating Th2 cytokines, GATA-3, STAT6, and Foxp3 transcription factors in an asthmatic mouse model. Environmental Toxicology and Pharmacology. Environ Toxicol Pharmacol 52: 99-113.

DOI:10.1016/j.etap.2017.03.011.

28. Kinsella, J. P., A. Greenough, and S. H. Abman. 2006. Bronchopulmonary dysplasia. Lancet 367: 1421-1431. DOI:10.1016/S0140-6736(06)68615-7.

29. Kotecha, S. 2000. Lung growth: implications for the newborn infant. Arch Dis Child Fetal Neonatal Ed 82 (1): F69-F74. DOI:10.1136/fn.82.1.f69.

30. Jensen, E. A., and B. Schmidt. 2014. Epidemiology of bronchopulmonary dysplasia. Birth Defects Res A Clin Mol Teratol 100: 145-157. DOI:10.1002/bdra.23235.

31. Lewandowski, A. J., W. M. Bradlow, D. Augustine, E. F. Davis, J. Francis, A. Singhal, A. Lucas, S. Neubauer, K. McCormick, and P. Leeson. 2013. Right ventricular systolic dysfunction in young adults born preterm. Circulation 128 (7): 713-720. DOI:10.1161/CIRCULATIONAHA.113.002583. 
32. Liao, J., V. S. Kapadia, L. S. Brown, N. Cheong, C. Longoria, D. Mija, M. Ramgopal, J. Mirpuri, D. C. McCurnin, and R. C. Savani: The NLRP3 inflammasome is critically involved in the development of bronchopulmonary dysplasia. Nat Commun, 2015, 6: 8977. DOI:10.1038/ ncomms9977.

33. Lignelli, E., F. Palumbo, D. Myti, and R. E. Morty. 2019. Recent advances in our understanding of the mechanisms of lung alveolarization and bronchopulmonary dysplasia. Am J Physiol Lung Cell Mol Physio/317: L832-L887. DOI:10.1152/ajplung.00369.2019.

34. Joshi, S., and S. Kotecha. 2007. Lung growth and development. Early Hum Dev 83 (12): 789-794. DOI:10.1016/j.earlhumdev.2007.09.007.

35. Morty, R. E. 2018. Recent advances in the pathogenesis of BPD. Semin Perinatol 42 (7): 404-412. DOI:10.1053/j.semperi.2018.09.001.

36. Nakanishi, H., A. Uchiyama, and S. Kusuda. 2016. Impact of pulmonary hypertension on neurodevelopmental outcome in preterm infants with bronchopulmonary dysplasia: a cohort study. $J$ Perinatol 36 (10): 890-896. DOI:10.1038/jp.2016.108.

37. Nardiello, C., I. Mižíková, and R. E. Morty. 2017. Looking ahead: where to next for animal models of bronchopulmonary dysplasia? Cell Tissue Res 367 (3): 457-468. DOI:10.1007/s00441-016-2534-3.

38. Negi, R., D. Pande, A. Kumar, R. S. Khanna, and H. D. Khanna. 2012. Evaluation of biomarkers of oxidative stress and antioxidant capacity in the cord blood of preterm low birth weight neonates. $J$ Matern Fetal Neonatal Med 25 (8): 1338-1341. DOI:10.3109/14767058.2011.633672.

39. O'Reilly, M., and B. Thebaud: Animal models of bronchopulmonary dysplasia. The term rat models. Am J Physiol Lung Cell Mol Physiol, 2014,307: L948-L958. DOl:10.1152/ajplung. 00160.2014.

40. Ozsurekci, Y., and K. Aykac: Oxidative Stress Related Diseases in Newborns. Oxid Med Cell Longev, 2016(undefined), 2768365. DOI:10.1155/2016/2768365.

41. Pakvasa, M. A., V. Saroha, and M. Patel Ravi. 2018. Optimizing Caffeine Use and Risk of Bronchopulmonary Dysplasia in Preterm Infants: A Systematic Review, Meta-analysis, and Application of Grading of Recommendations Assessment, Development, and Evaluation Methodology. Clin Perinatol 45 (2): 273-291. DOI:10.1016/j.clp.2018.01.012.

42. Pastorino, G., L. Cornara, S. Soares, F. Rodrigues, and M. B. P. P. Oliveira. 2018. Liquorice (Glycyrrhiza glabra): A phytochemical and pharmacological review. Phytother Res 32: 2323-2339.

DOI:10.1002/ptr.6178.

43. Peng, D., J. Li, Y. Deng, X. Zhu, L. Zhao, Y. Zhang, Z. Li, S. Ou, S. Li, and Y. Jiang. 2020. Sodium paraaminosalicylic acid inhibits manganese-induced NLRP3 inflammasome-dependent pyroptosis by inhibiting NF-кB pathway activation and oxidative stress. J Neuroinflammation 17 (1): 343. DOI:10.1186/s12974-020-02018-6.

44. Perrone, S., C. Bracciali, N. Di Virgilio, and G. Buonocore. 2016. Oxygen Use in Neonatal Care: A Twoedged Sword. Front Pediatr 4 (undefined): 143. DOI:10.3389/fped.2016.00143.

45. Poggi, C., and C. Dani: Antioxidant strategies and respiratory disease of the preterm newborn: an update. Oxid Med Cell Longev, 2014(undefined), 721043. DOI:10.1155/2014/721043. 
46. Rosanna, D. P., and C. Salvatore. 2012. Reactive oxygen species, inflammation, and lung diseases. Curr Pharm Des 18 (26): 3889-3900. DOI:10.2174/138161212802083716.

47. Principi, N., G. Di Pietro, and S. Esposito. 2018. Bronchopulmonary dysplasia: clinical aspects and preventive and therapeutic strategies. J Trans/ Med 16: 36. DOI:10.1186/s12967-018-1417-7.

48. Ryan, R. M., Q. Ahmed, and S. Lakshminrusimha: Inflammatory mediators in the immunobiology of.

49. bronchopulmonary dysplasia. Clin Rev Allergy Immunol, 2008, 34:174-190. DOI:10.1007/s 12016007-8031-4.

50. Sanchez-Solis, M., P. W. Garcia-Marcos, J. Agüera-Arenas, P. Mondejar-Lopez, and L. Garcia-Marcos. 2020. Impact of early caffeine therapy in preterm newborns on infant lung function. Pediatr Pulmonol 55 (1): 102-107. DOI:10.1002/ppul.24540.

51. Saugstad Ola, D. 2018. Oxygenation of the Immature Infant: A Commentary and Recommendations for Oxygen Saturation Targets and Alarm Limits. Neonatology 114 (1): 69-75. DOI:10.1159/000486751.

52. Savani, R. C.: Modulators of Inflammation in Bronchopulmonary Dysplasia.Seminars in Perinatology, 2018, 42(7), 459-470. DOI: https://doi.org/10.1053/j.semperi.2018.09.009.

53. Sipola-Leppänen, M., M. Vääräsmäki, M. Tikanmäki, P. Hovi, S. Miettola, A. Ruokonen, A. Pouta, M. Järvelin, and E. Kajantie. 2014. Cardiovascular risk factors in adolescents born preterm. Pediatrics 134 (4): e1072-e1081. DOI:10.1542/peds.2013-4186.

54. Sriram, S., M. D. Schreiber, M. E. Msall, K. C. K. Kuban, R. M. Joseph, T. M. O' Shea, E. N. Allred, and A. Leviton, ELGAN Study Investigators: Cognitive Development and Quality of Life Associated With BPD in 10-Year-Olds Born Preterm. Pediatrics. 2018. 141(6), undefined. DOI:10.1542/peds.2017-2719.

55. Stoll, B. J., N. I. Hansen, E. F. Bell, S. Shankaran, A. R. Laptook, M. C. Walsh, E. C. Hale, N. S. Newman, K. Schibler, and W. A. Carlo, et al. 2010. Neonatal outcomes of extremely preterm infants from the NICHD neonatal research network. Pediatrics 126: 443-456. DOI:10.1542/peds.2009-2959.

56. Stoll, B. J., N. I. Hansen, E. F. Bell, M. C. Walsh, W. A. Carlo, S. Shankaran, A. R. Laptook, P. J. Sanchez, K. P. Van Meurs, and M. Wyckoff, et al.: Trends in care practices, morbidity, and mortality of extremely preterm neonates, 1993-2012. JAMA,2015,314: 1039-1051. DOI:10.1001/jama.2015.10244.

57. Swanson, K. V., M. Deng, and J. P. Y. Ting. 2019. The NLRP3 inflammasome: molecular activation and regulation to therapeutics. Nat Rev Immunol 19 (8): 477-489. DOI:10.1038/s41577-019-0165-0.

58. Sweet, D. G., V. Carnielli, G. Greisen, M. Hallman, E. Ozek, A. Te Pas, R. Plavka, C. C. Roehr, D. Saugstad Ola, and U. Simeoni, et al. 2019. European Consensus Guidelines on the Management of Respiratory Distress Syndrome - 2019 Update. Neonatology 115 (4): 432-450. DOI:10.1159/000499361.

59. Teng, J., Q. Mei, X. Zhou, Y. Tang, R. Xiong, W. Qiu, R. Pan, Y. Law Betty, K. Wong Vincent, and L. Yu Chong, et al.: Polyphyllin VI Induces Caspase-1-Mediated Pyroptosis via the Induction of ROS/NFKB/NLRP3/GSDMD Signal Axis in Non-Small Cell Lung Cancer. Cancers (Basel), 2020, 12(1), undefined. DOI:10.3390/cancers12010193. 
60. Wang, J., and W. Dong. 2018. Oxidative stress and bronchopulmonary dysplasia. Gene 678 (undefined): 177-183. DOl:10.1016/j.gene.2018.08.031.

61. Wei, Z., G. Nie, F. Yang, S. Pi, C. Wang, H. Cao, X. Guo, P. Liu, G. Li, G. Hu, and C. Zhang. 2020. Inhibition of ROS/NLRP3/Caspase-1 mediated pyroptosis attenuates cadmium-induced apoptosis in duck renal tubular epithelial cells. Environ Pollut 273 (undefined): 115919.

DOI:10.1016/j.envpol.2020.115919.

62. Wilson-Costello, D., M. C. Walsh, J. C. Langer, R. Guillet, A. R. Laptook, B. J. Stoll, S. Shankaran, N. N. Finer, M. K. P. Van, and W. A. Engle, et al. 2009. Impact of postnatal corticosteroid use on neurodevelopment at 18 to 22 months' adjusted age: effects of dose, timing, and risk of bronchopulmonary dysplasia in extremely low birth weight infants. Pediatrics 123 (3): e430-e437. DOI:10.1542/peds.2008-1928.

63. Wright, C. J., F. Agboke, F. Chen, P. La, G. Yang, and P. A. Dennery. 2010. Nitric oxide inhibits hyperoxia-induced NF-B activation in neonatal pulmonary microvascular endothelial cells. Pediatr Res 68 (6): 484-489. DOI:10.1203/PDR.0b013e3181f917b0.

64. Wright, C. J., and H. Kirpalani: Targeting inflammation to prevent bronchopulmonary dysplasia: can new insights be translated into therapies?. Pediatrics. 2011. 128: 111 - 26. DOI:10.1542/ peds.2010-3875.

65. Yang, Y., H. Wang, M. Kouadir, H. Song, and F. Shi. 2019. Recent advances in the mechanisms of NLRP3 inflammasome activation and its inhibitors. Cell Death Dis 10 (2): 128. DOI:10.1038/s41419019-1413-8.

66. Zha, L., J. Chen, S. Sun, L. Mao, X. Chu, H. Deng, J. Cai, X. Li, Z. Liu, and W. Cao. 2014. Soyasaponins can blunt inflammation by inhibiting the reactive oxygen species-mediated activation of PI3K/Akt/NF-kB pathway. PLoS One 9 (9): e107655. DOI:10.1371/journal.pone.0107655.

67. Zhang, M., Z. Chang, F. Zhao, P. Zhang, Y. J. Hao, L. Yan, N. Liu, J. L. Wang, L. Bo, P. Ma, W. Zhou, X. Ma, Q. B. Xu, and R. Zhou. 2019. Protective Effects of 18ß-Glycyrrhetinic Acid on MonocrotalineInduced Pulmonary Arterial Hypertension in Rats. Frontiers in Pharmacology 10: 13.

DOI:10.3389/fphar.2019.00013.

68. Zhong, Z., Y. Zhai, S. Liang, Y. Mori, R. Han, F. S. Sutterwala, and L. Qiao. 2013. TRPM2 links oxidative stress to NLRP3 inflammasome activation. Nat Commun 4 (undefined): 1611.

DOI:10.1038/ncomms2608.

\section{Figures}



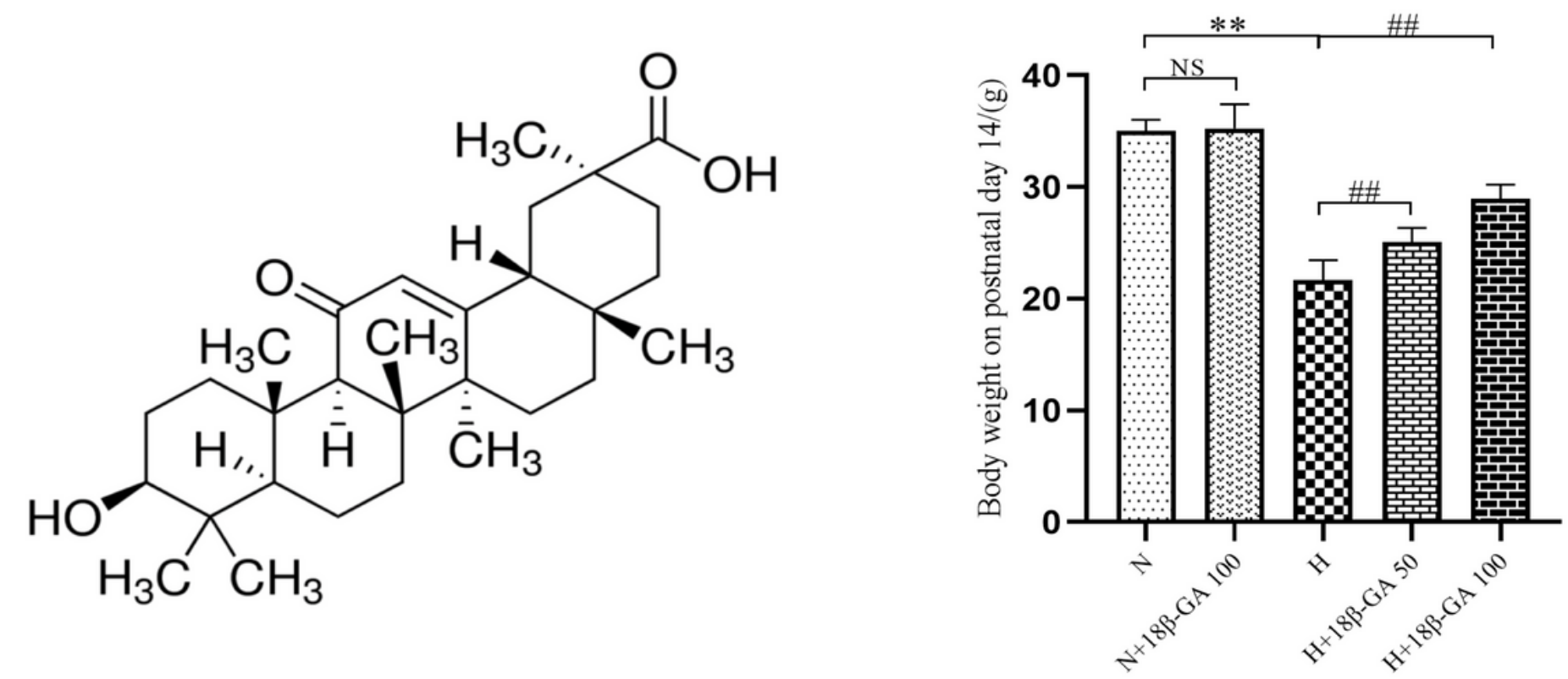

Figure 1

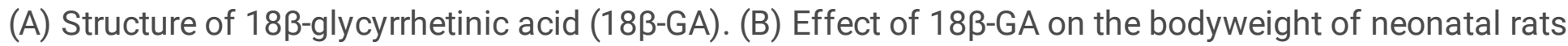
on $\mathrm{PN14}$. The values are the mean \pm standard deviation. NS not significant; $* \star P<0.01$ vs. $N$ group; \#\# $P$ $<.01$ vs. H group.

\section{Figure 2}

Effect of $18 \beta-G A$ on alveolarization in neonatal rats. $18 \beta-G A$ treatment improved the alveolar simplification induced by hyperoxia exposure. Hematoxylin and eosin (HE) staining of lung tissue slides from each group (light microscopy, 100x magnification; scale bar, $200 \mu \mathrm{m}$ ). Alveolarization was quantified by RAC value. The values are the mean \pm standard deviation. NS not significant; ** $P<0.01$ vs. N group; \#\# $P<0.01$ vs. H group. 

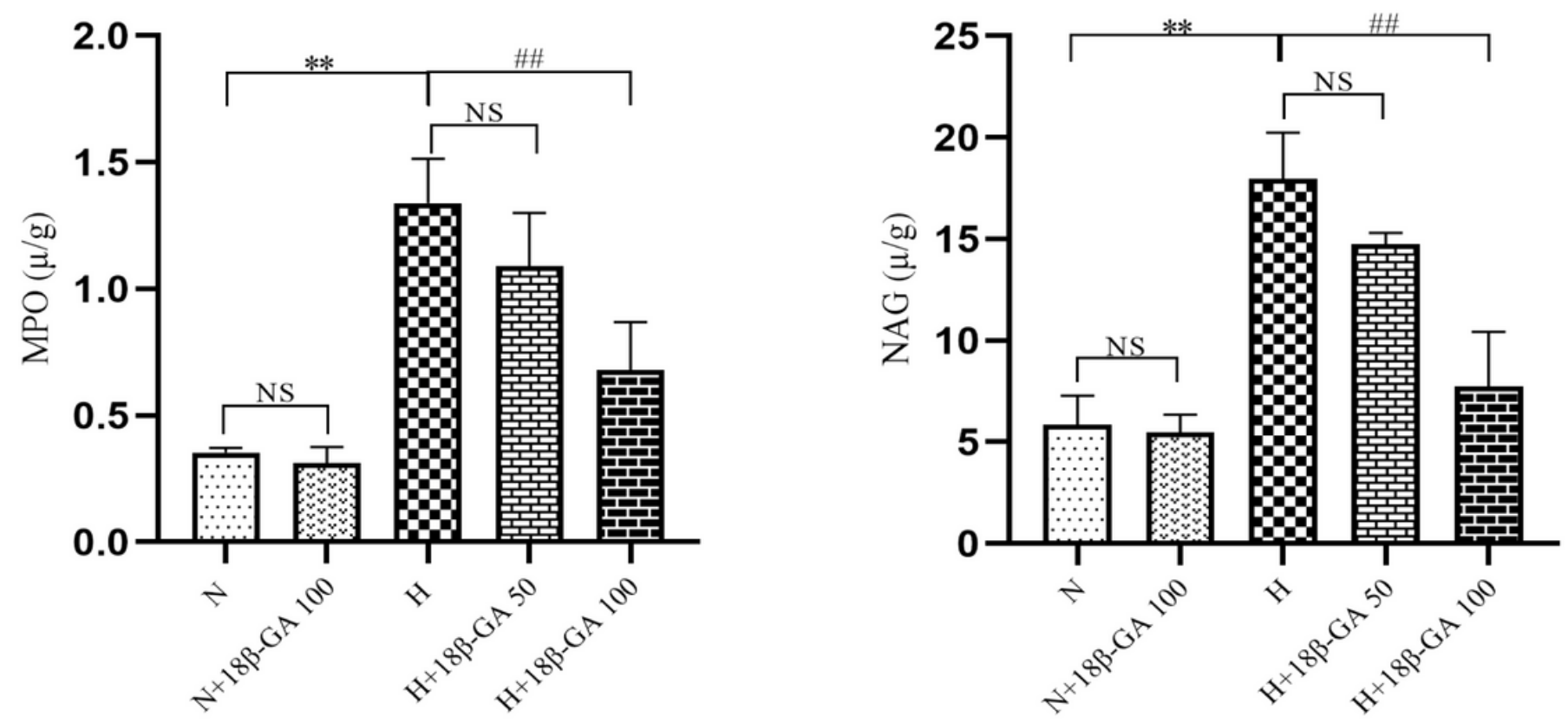

Figure 3

Effects of $18 \beta-G A$ on the inflammatory cells infiltration in lung tissues. 18ß-GA decreased myeloperoxidase (MPO) (A) and N-Acetyl- $\beta$-D-glucosaminidase (NAG) (B) activities in lung homogenates of rats exposed to hyperoxia conditions. The values are the mean \pm standard deviation. NS not significant; ** $\mathrm{P}<0.01$ vs. $\mathrm{N}$ group; \#\# $\mathrm{P}<0.01$ vs. H group.

A

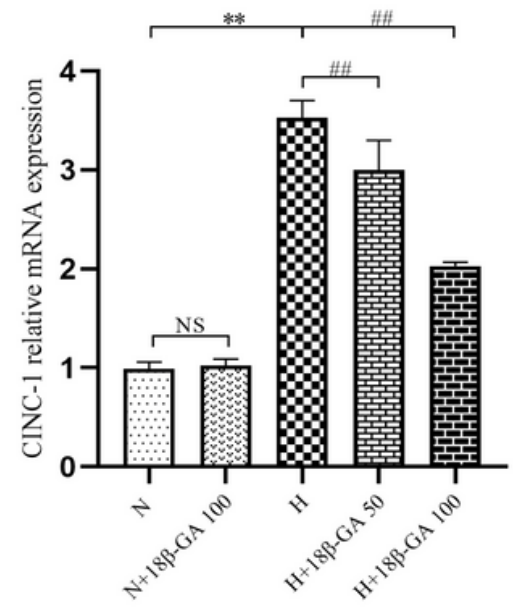

$\mathrm{B}$

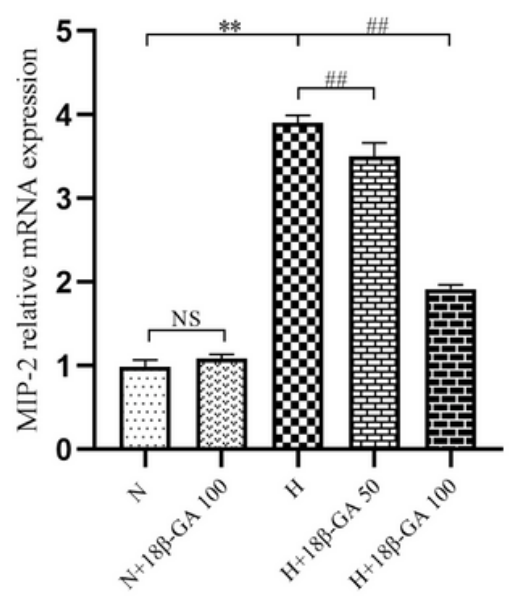

$\mathrm{C}$

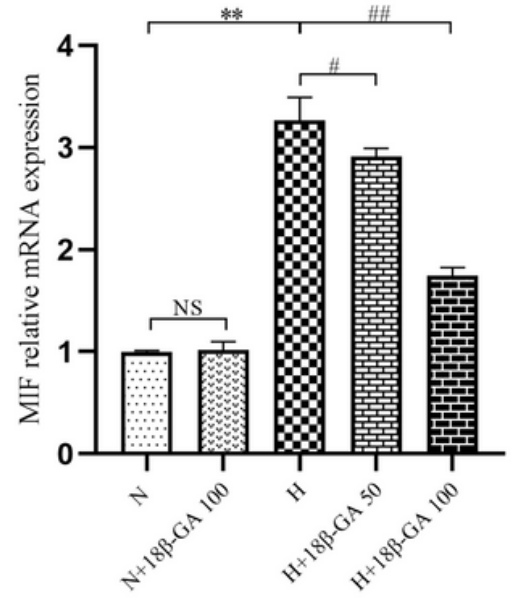

Figure 4

Effects of 18ß-GA on the chemokines in lung tissues. 18ß-GA decreased cytokine-induced neutrophil chemoattractant-1 (CINC-1), macrophage inflammatory protein-2 (MIP-2), and macrophage migration 
inhibitory factor (MIF) in lung homogenates of rats exposed to hyperoxia conditions. The values are the mean \pm standard deviation. NS not significant; ${ }^{\star \star} \mathrm{P}<0.01$ vs. N group; \#\# $\mathrm{P}<0.01$ vs. H group.

A

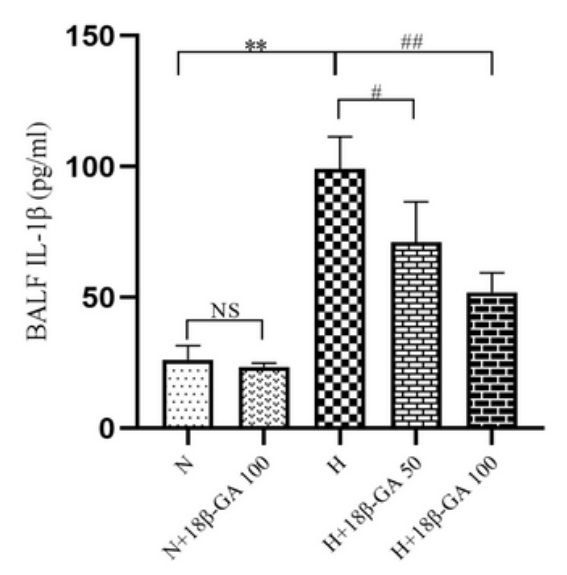

B

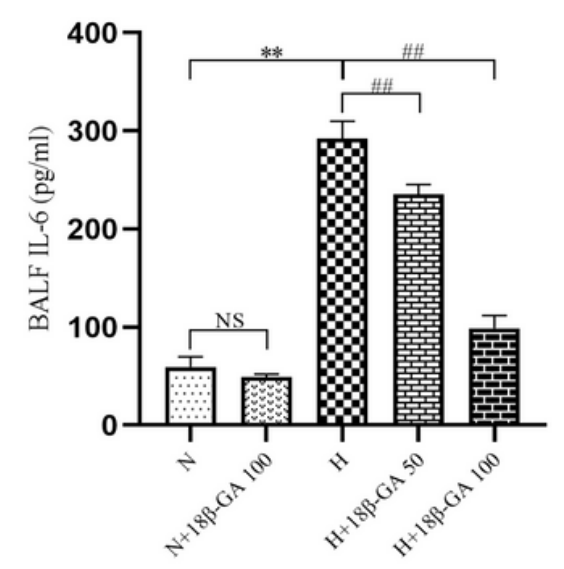

C

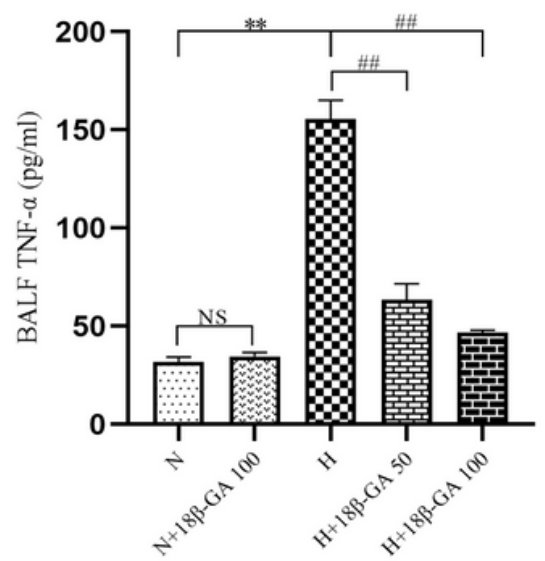

Figure 5

Effects of $18 \beta-G A$ on the expression of pro-inflammatory cytokines in bronchoalveolar lavage fluid (BALF). 18 $\beta-G A$ reduced IL-1 $\beta$ (A), IL-6 (B), and TNF- $\alpha(C)$ expression in BALF of rats exposed to hyperoxia. The values are the mean \pm standard deviation. NS not significant; ${ }^{\star *} \mathrm{P}<0.01$ vs. $\mathrm{N}$ group; \#\# $\mathrm{P}$ $<0.01$ vs. H group. 


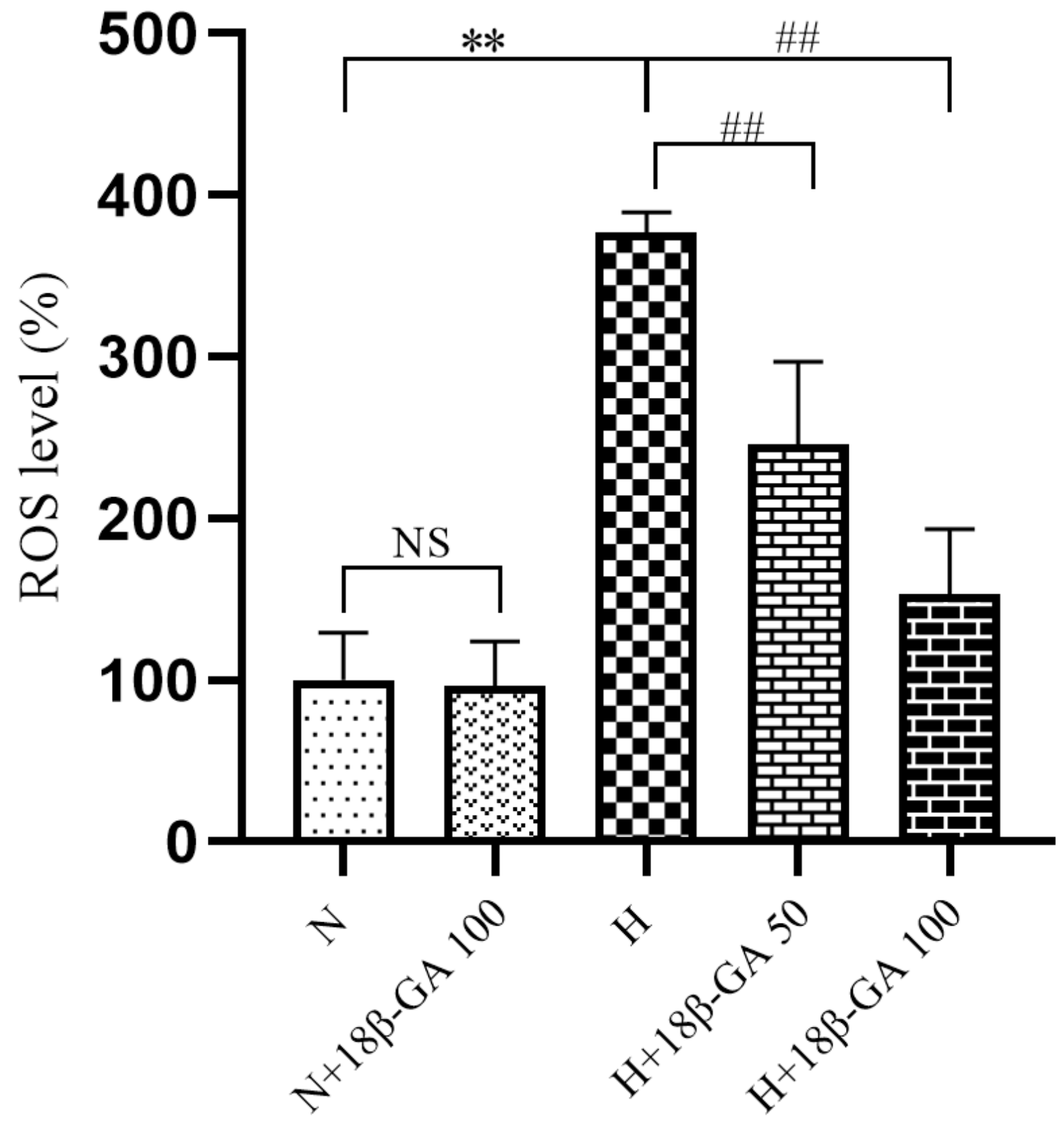

Figure 6

Effect of $18 \beta-G A$ on the ROS production in rats lung. 18ß-GA treatment decreased the ROS level of rats lung with hyperoxia exposure. The values are the mean \pm standard deviation. NS not significant; $* \star \mathrm{P}<$ 0.01 vs. $\mathrm{N}$ group; \#\# $\mathrm{P}<0.01$ vs. $\mathrm{H}$ group. 
A
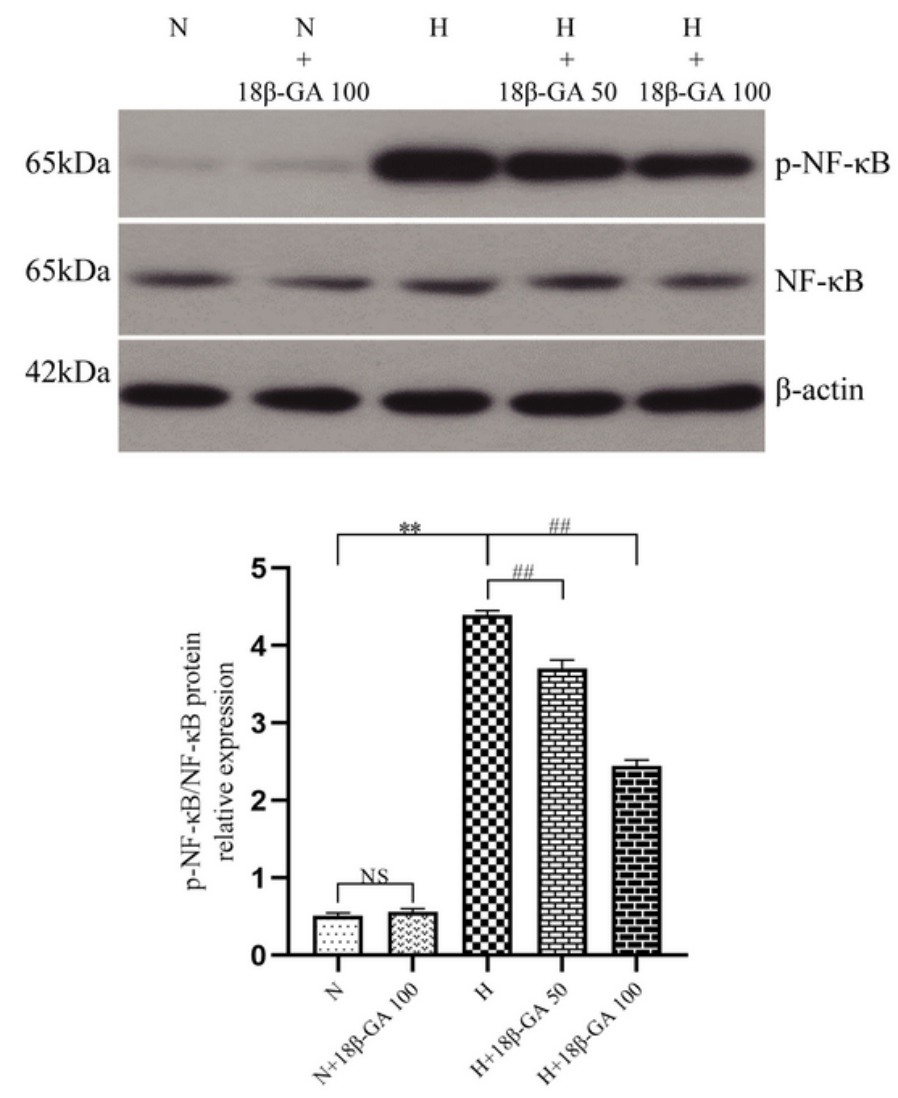

B
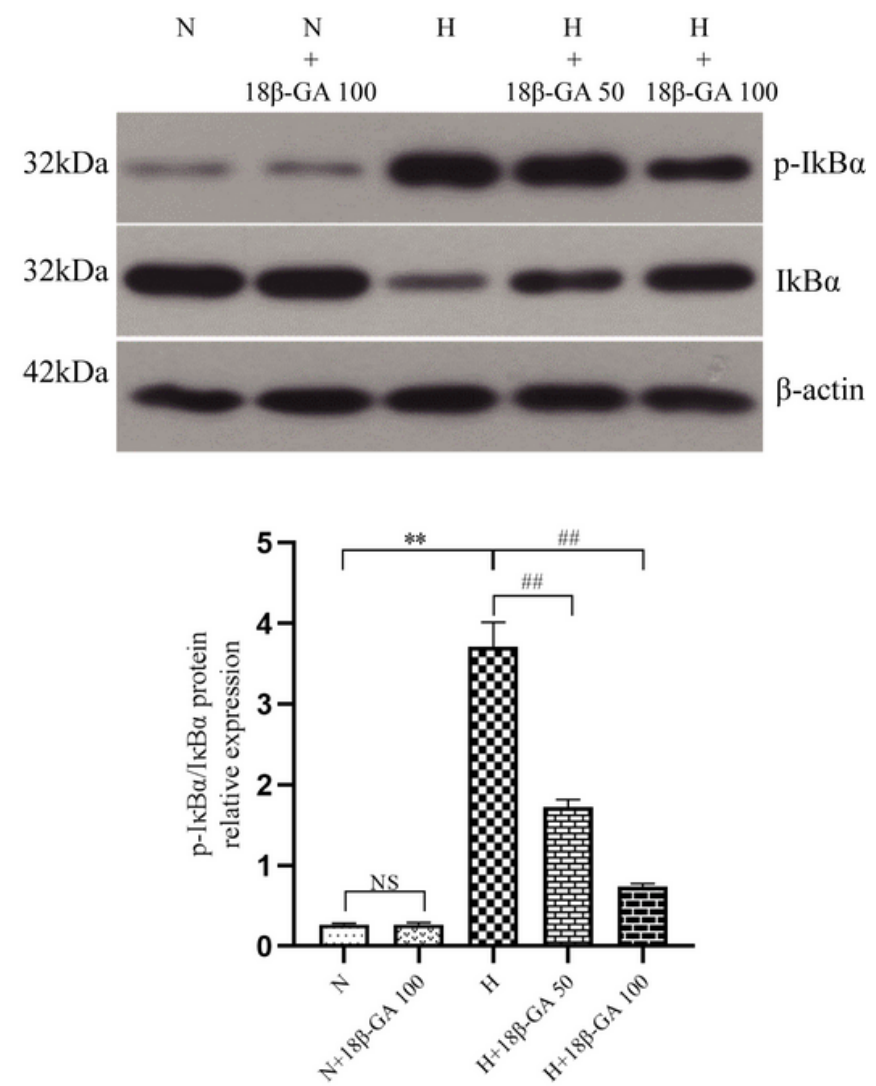

Figure 7

Effects of $18 \beta-G A$ on the NF-KB pathway in rat lungs. $18 \beta-G A$ reduced NF-KB pathway activation in rats exposed to hyperoxia. (A) Representative image and semiquantitative analysis of phosphor-NF-KB ( $\mathrm{p}-\mathrm{NF}-$ $\mathrm{KB}$ ) and NF-KB protein expression in lung homogenates. (B) Representative image and semiquantitative analysis of phosphor-IкBa $\left(\mathrm{p}-\mathrm{I}_{\mathrm{KBa}}\right)$ and $\mathrm{I}_{\mathrm{KBa}}$ protein expression in lung homogenates. The values are the mean \pm standard deviation. NS not significant $* \star P<0.01$ vs. N group; \#\# $P<0.01$ vs. $H$ group. 
A
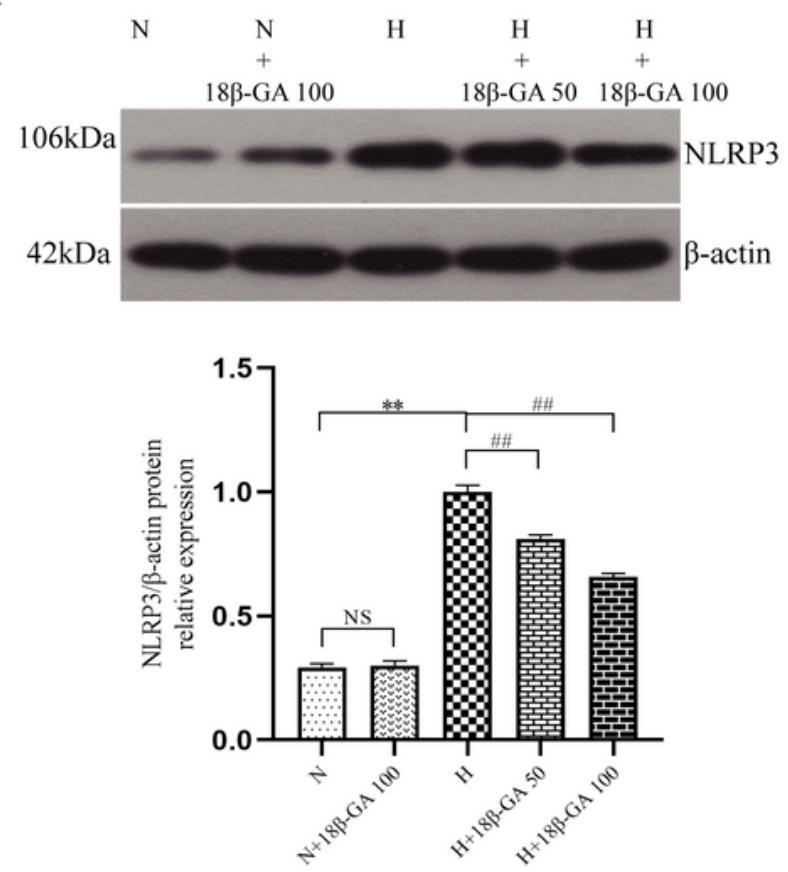

C
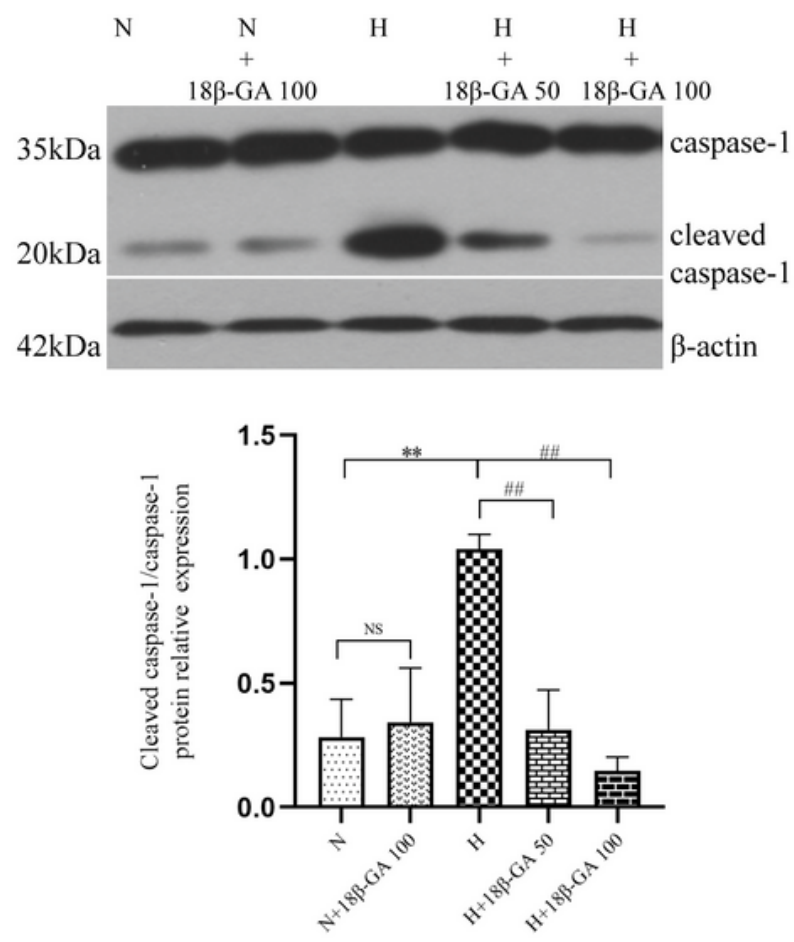

B
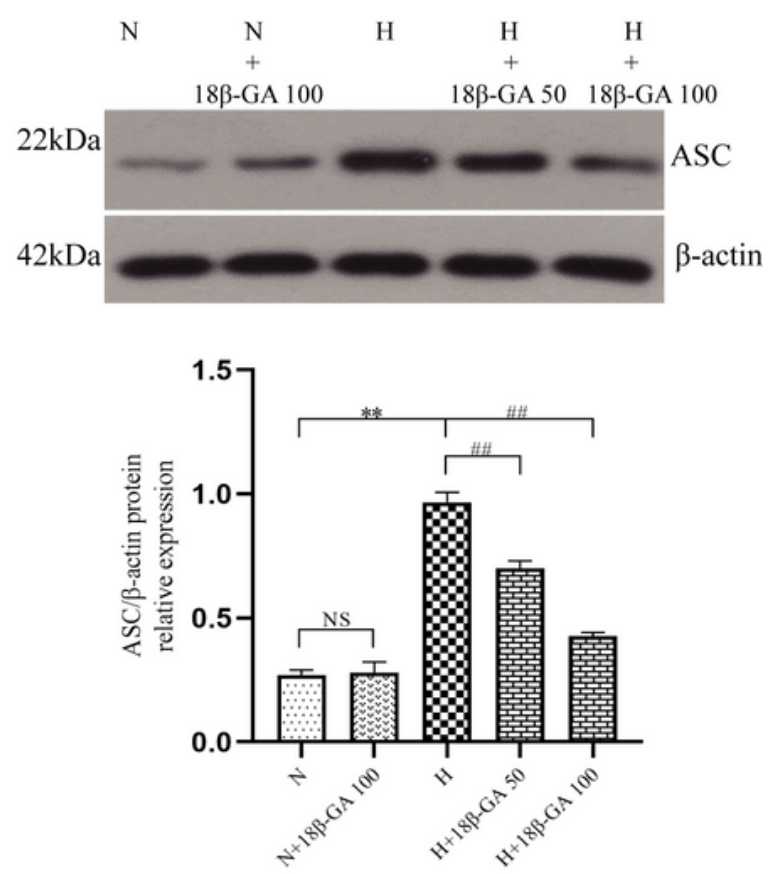

D 
1 protein expression in lung tissue homogenates. (D) Caspase- 1 activity in lung tissue homogenates. The values are the mean \pm standard deviation. NS not significant $* * P<0.01$ vs. $N$ group; \#\# $P<0.01$ vs. H group.
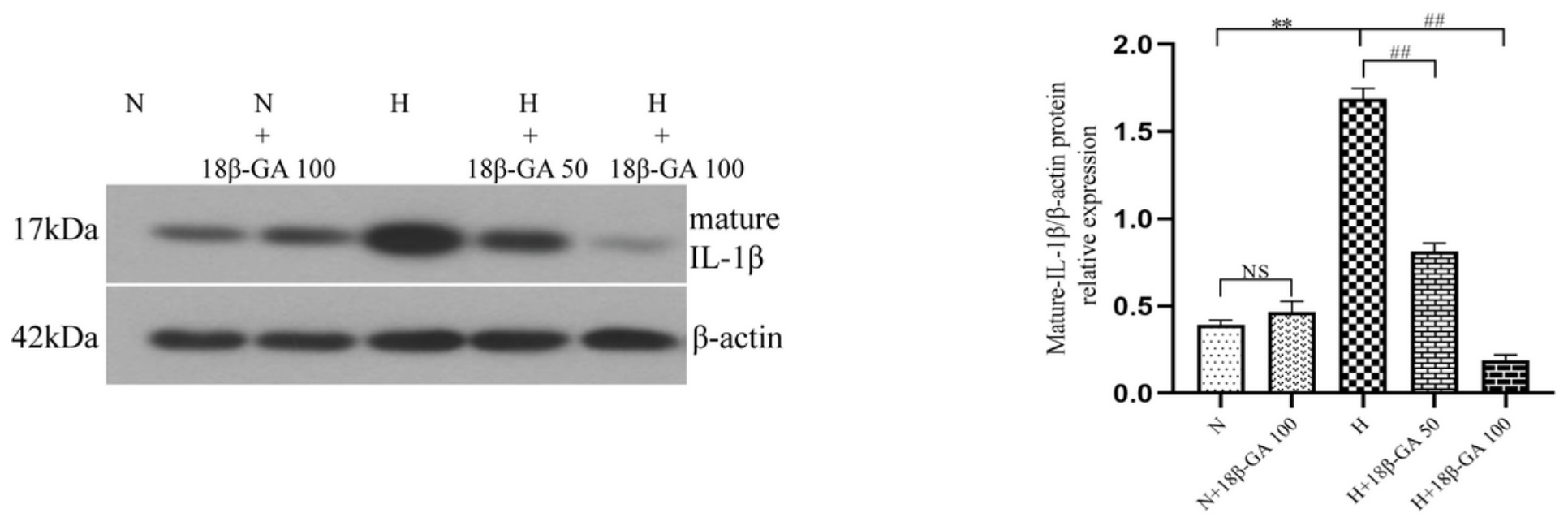

Figure 9

Effects of $18 \beta-G A$ on the mature IL-1 $1 \beta .18 \beta-G A$ decreased the protein expression of mature IL-1 $\beta$ in rats exposed to hyperoxia conditions. Representative image and semiquantitative analysis of mature IL-1 $\beta$ protein expression in lung tissue homogenates. The values are the mean \pm standard deviation. NS not significant $* * P<0.01$ vs. $N$ group; \#\# $P<0.01$ vs. H group. 